\title{
Abiraterone in Metastatic Salivary Duct Carcinoma
}

\author{
Damien Urban, MBBS, BMedSc ${ }^{\text {a }}$; Danny Rischin, MD, FRACPa; Christopher Angel, FRCPA ${ }^{\text {; }}$; \\ Ieta D'Costa, FRANZCR c; and Benjamin Solomon, PhD, FRACPa
}

\begin{abstract}
Salivary duct carcinoma (SDC) is a rare, aggressive salivary gland malignancy with limited evidence guiding standard treatment. SDC is known to overexpress the androgen receptor, with only a handful of cases reporting responses to androgen blockade. This report presents a case of SDC responding to multiple lines of androgen blockade, including a rapid response to abiraterone, a CYP17 inhibitor effective in prostate cancer. This case represents the first published report of SDC responding to abiraterone and illustrates that androgen receptor expressing SDC may be treated with multiple lines of androgen blockade, including newer agents such as abiraterone. This case suggests that SDC may continue to be androgen-dependent after progression on androgen deprivation, which is analogous to prostate cancer. (J Natl Compr Canc Netw 2015;13:288-290)
\end{abstract}

Salivary duct carcinoma (SDC) is a rare cancer representing less than $10 \%$ of all salivary gland malignancies. ${ }^{1,2}$ It is an aggressive tumour, with many patients succumbing to disseminated disease. ${ }^{3}$ Given its rarity, no standard treatment exists for metastatic SDC.

Androgen receptors (ARs) are overexpressed in most SDCs, and reports have shown some activity with anti-androgen therapy using bicalutamide with or without goserelin. ${ }^{4}$ Abiraterone, by inhibiting adrenal and testicular androgen synthesis through CYP17, has re-

From the Departments of a Medical Oncology, 'bathology, and 'Radiation Oncology, Peter MacCallum Cancer Centre, East Melbourne, Australia.

Submitted June 10, 2014; accepted for publication

December 15, 2014.

The authors have disclosed that they have no financial interests, arrangements, affiliations, or commercial interests with the manufacturers of any products discussed in this article or their competitors.

Correspondence: Damien Urban, MBBS, BMedSc, Department of Medical Oncology, Peter MacCallum Cancer Centre, St Andrews

Place, East Melbourne, Australia 3002.

E-mail: damien.urban@sheba.health.gov.il cently shown significant efficacy in castration-resistant prostate cancer, ${ }^{5,6}$ but reports of its use in SDC have not been published.

This report describes what seems to be the first published clinical and radiologic response to abiraterone in a patient with metastatic SDC refractory to bicalutamide and goserelin.

\section{Case Report}

A previously healthy, 45-year-old man with no smoking history presented in October 2011 with a right-sided neck mass. Imaging studies, including a CT and fluorodeoxyglucose (FDG)-PET/CT, showed enlarged level II and V right neck nodes with a lesion in the right parotid gland. An open biopsy of a lymph node confirmed adenocarcinoma.

Subsequently, the patient underwent a right parotidectomy and modified neck dissection. Pathologic assessment showed an SDC within the parotid gland with extracapsular extension, positive margins, and 57 of 57 lymph nodes involved. Diagnosis was confirmed with immunohistochemical stains being positive for GCDFP-15, CK7, and AR, and negative staining for c-kit, CK20, and HER2.

The patient received 60 Gy of postoperative radiotherapy in 30 fractions and then adjuvant chemotherapy with cyclophosphamide, doxorubicin, and cisplatin (CAP). During the third cycle of chemotherapy, a palpable contralateral lymph node appeared and fine-needle aspiration cytology confirmed recurrence. An FDG-PET/CT demonstrated that this left cervical lymph node and an additional sclerotic left iliac bone metastasis. Anti-androgen therapy was initiated with goserelin, and a short-term partial metabolic response of 4 months' duration was shown by 
FDG-PET/CT. At progression, combined androgen deprivation with the addition of bicalutamide provided a good metabolic response for a further 10 months. At this time, PET/CT scan revealed progressive disease in the neck and skeleton, and a repeat biopsy confirmed persistent strong AR expression (Figure 1).

Extrapolating from recent prostate cancer trials, abiraterone at a dosage of $1000 \mathrm{mg} / \mathrm{d}$ with prednisone, $5 \mathrm{mg}$ twice daily (with ongoing goserelin) was commenced, and within 3 weeks a complete clinical response was seen within the cervical neck lymph node. A PET/CT scan at 3 months confirmed resolution of FDG uptake in the neck (Figure 2) and a significant favorable metabolic response in the skeletal metastases. At 6 months, PET/CT confirmed ongoing response. After 10 months on abiraterone and goserelin, increasing pain in the sacrum was described and a repeat PET/CT confirmed progressive osteoblastic bone disease with ongoing control of the neck disease. Until this point, no radiotherapy was delivered to bone metastases. Abiraterone was well tolerated with no unexpected side effects.

\section{Discussion}

Salivary gland malignancies are diagnosed in 1.3 per 100,000 persons, constituting $12.0 \%$ of oral cavity/ pharyngeal malignancies and less than $0.5 \%$ of all cancers in the United States. ${ }^{7}$ Salivary gland carcinomas are divided into 24 different entities by the WHO, including mucoepidermoid, adenoid cystic carcinoma, adenocarcinoma, and SDC, with the last accounting for up to $9 \%$ of salivary gland malignancies. Nuclear reactivity for ARs has been reported to occur in more than $40 \%$ to $93 \%$ of SDCs. ${ }^{8,9}$ In fact, the WHO maintains that all SDCs show strong nuclear reactivity for ARs. ${ }^{1}$

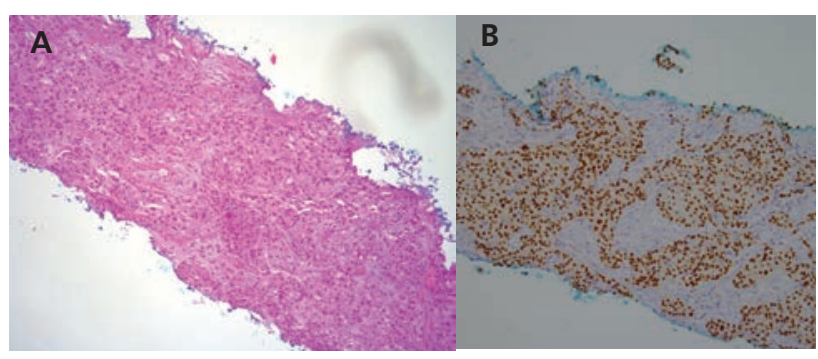

Figure 1 Histologic features of salivary duct carcinoma. (A) Hematoxylin-eosin staining. (B) Androgen receptor immunohistochemistry showing strong positive reaction in tumor cells.
Given the rarity of SDC and the resultant lack of high-quality evidence, no standard treatment guidelines exist. Evidence suggests that SDC is associated with potentially targetable molecular abnormalities, including AR expression, ${ }^{10}$ HER2 overexpression, ${ }^{11}$ and, rarely, EGFR mutations, ${ }^{12}$ PIK3CAactivating, or BRAF-activating mutations. ${ }^{13}$ However, responses to specific molecular targeting is limited to single or few case reports.

It has been suggested that SDC may be similar to prostate cancer with respect to androgen dependence and to breast cancer with respect to histology and HER2 overexpression ${ }^{9,12,14}$ Given the rarity of SDC, treatment options for SDC may be inferred from both these cancers. Responses to trastuzumab ${ }^{11}$ and androgen deprivation therapy in SDC have been described in case reports ${ }^{14-16}$ and a case series. ${ }^{4}$ The case series described 2 partial responses and 3 patients with stable disease among 10 patients (clinical benefit of 50\%) with AR-positive SDC treated with androgen deprivation. Nine of 10 patients received single-agent bicalutamide as first-line therapy, with 3 patients receiving second-line androgen deprivation therapy with the addition of goserelin. However, no radiologic responses were reported in patients receiving second-line therapy. Because of the limited number of reports of androgen deprivation in ARexpressing SDC, the most effective strategy to inhibit the androgen pathway in SDC is unknown.

It has been established that castration-resistant prostate cancer remains dependent on androgen stimulation for survival and progression. ${ }^{17}$ Furthermore, enzymes involved in the synthesis of androgens, including CYP17, are overexpressed in prostate cancer. ${ }^{18}$ Abiraterone, an irreversible inhibitor of CYP17, thereby inhibits the synthesis of andro-

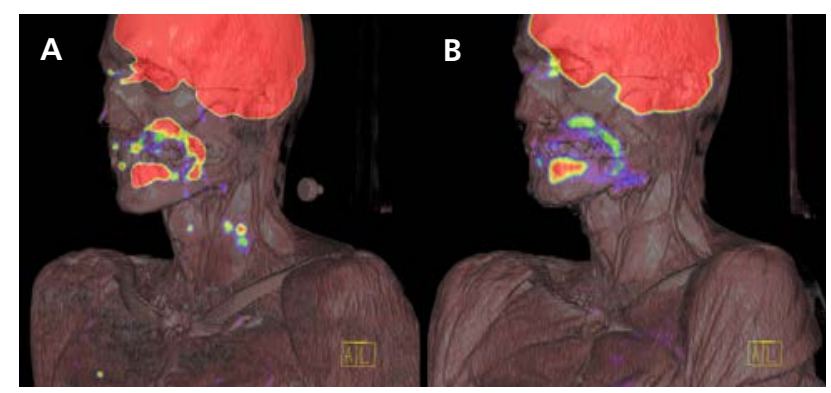

Figure 2 PET/CT reconstruction demonstrating a complete metabolic response to abiraterone in cervical nodal disease. (A) Recurrent cervical neck involvement after 2 previous lines of androgen deprivation therapy (May 21, 2013). (B) Complete metabolic response in the cervical nodal disease after 3 months of treatment with abiraterone (September 23, 2013). The metabolic activity in the tongue is physiologic. 
Urban et al

gens in the testes and adrenal glands, and in the tumor itself. It has recently shown significant activity in castration-resistant prostate cancer, with an improvement in overall survival in patients previously treated with chemotherapy, ${ }^{5}$ and an increase in progression-free survival in chemotherapy-naïve patients. ${ }^{6}$ The 10 -month progression-free survival seen in the present patient while on abiraterone is remarkably similar to the median time to prostatespecific antigen progression of 10.5 months in castration-resistant prostate cancer. ${ }^{5}$

The common side effects of abiraterone include mineralocorticoid effects caused by a compensatory increase in adrenocorticotropic hormone (ACTH), including hypertension, hypokalemia, and fluid retention, and, uncommonly, hepatotoxicity with an increase in bilirubin and/or hepatic transaminases. ${ }^{5}$ Prednisone is prescribed together with abiraterone to prevent the compensatory increase in ACTH, and caution should be taken in patients with cardiovascular or hepatic disease. ${ }^{5}$

\section{Conclusions}

Patient response in the present case to multiple lines of androgen blockade, including abiraterone, suggests that androgen dependence also occurs in AR-expressing SDC, and that continued blockade of this pathway despite progression may be beneficial. Consequently, it may be reasonable to consider other inhibitors of the androgen axis that have been proven effective in prostate cancer, such as enzalutamide, in the treatment of this disease entity. In the absence of prospective clinical data, multiple lines of androgen deprivation therapy should be considered in patients with AR-expressing SDC, but the appropriate sequence of anti-androgen therapies is unknown. If feasible and when recruitment begins, preference should be given to enrollment on a prospective clinical trial comparing chemotherapy versus anti-androgen therapy (ClinicalTrials.gov identifier: NCT01969578) in androgen-expressing salivary gland carcinomas.

\section{References}

1. Barnes L, Eveson JW, Reichart P, Sidransky D, eds. World Health Organisation Classification of Tumours. Pathology and Genetics of Head and Neck Tumours. Lyon, France: IARC Press; 2005.

2. Wang YL, Zhu YX, Chen TZ, et al. Clinicopathologic study of 1176 salivary gland tumors in a Chinese population: experience of one cancer center 1997-2007. Acta Otolaryngol 2012;132:879-886.

3. Guzzo M, Di Palma SD, Grandi C, Molinari R. Salivary duct carcinoma: clinical characteristics and treatment strategies. Head Neck 1997;19:126133.

4. Jaspers HCJ, Verbist BM, Schoffelen R, et al. Androgen receptor-positive salivary duct carcinoma: a disease entity with promising new treatment options. J Clin Oncol 2011;29:e473-476.

5. de Bono JS, Logothetis CJ, Molina A, et al. Abiraterone and increased survival in metastatic prostate cancer. N Engl J Med 2011;364:1995-2005.

6. Ryan CJ, Smith MR, de Bono JS, et al. Abiraterone in metastatic prostate cancer without previous chemotherapy. N Engl J Med 2013;368:138-148.

7. Howlader N, Noone AM, Krapcho M, et al, eds. SEER Cancer Statistics Review, 1975-2011, National Cancer Institute. Bethesda, MD. Available at: http://seer.cancer.gov/csr/1975_2011/, based on November 2013 SEER data submission, posted to the SEER web site, April 2014. Accessed February 6, 2015.

8. Locati LD, Perrone F, Losa M, et al. Treatment relevant target immunophenotyping of 139 salivary gland carcinomas (SGCs). Oral Oncol 2009;45:986-990.

9. Fan CY, Wang J, Barnes EL. Expression of androgen receptor and prostatic specific markers in salivary duct carcinoma: an immunohistochemical analysis of 13 cases and review of the literature. Am J Surg Pathol 2000;24:579-586.

10. Kapadia SB, Barnes L. Expression of androgen receptor, gross cystic disease fluid protein, and CD44 in salivary duct carcinoma. Mod Pathol 1998;11:1033-1038.

11. Krishnamurthy J, Krishnamurty DM, Baker JJ, et al. Salivary duct carcinoma responding to trastuzumab-based therapy: case report and review of the literature. Head Neck 2013;35:E372-375.

12. Williams MD, Roberts DB, et al. Genetic and expression analysis of HER2 and EGFR genes in salivary duct carcinoma: empirical and therapeutic significance. Clin Cancer Res 2010;16:2266-2274.

13. Nardi V, Sadow PM, Juric D, et al. Detection of novel actionable genetic changes in salivary duct carcinoma helps direct patient treatment. Clin Cancer Res 2013;19:480-490.

14. Locati LD, Quattrone P, Bossi P, et al. A complete remission with androgen-deprivation therapy in a recurrent androgen receptor-expressing adenocarcinoma of the parotid gland. Ann Oncol 2003;14:1327-1328.

15. Van der Hulst RW, van Krieken JH, van der Kwast TH, et al. Partial remission of parotid gland carcinoma after goserelin. Lancet 1994;344:817.

16. Soper MS, Iganej S, Thompson LD. Definitive treatment of androgen receptor-positive salivary duct carcinoma with androgen deprivation therapy and external beam radiotherapy. Head Neck 2014;36:E4-7.

17. Chen CD, Welsbie DS, Tran C, et al. Molecular determinants of resistance to antiandrogen therapy. Nat Med 2004;10:33-39.

18. Montgomery RB, Mostaghel EA, Vessella R, et al. Maintenance of intratumoral androgens in metastatic prostate cancer: a mechanism for castration-resistant tumor growth. Cancer Res 2008;68:4447-4454. 